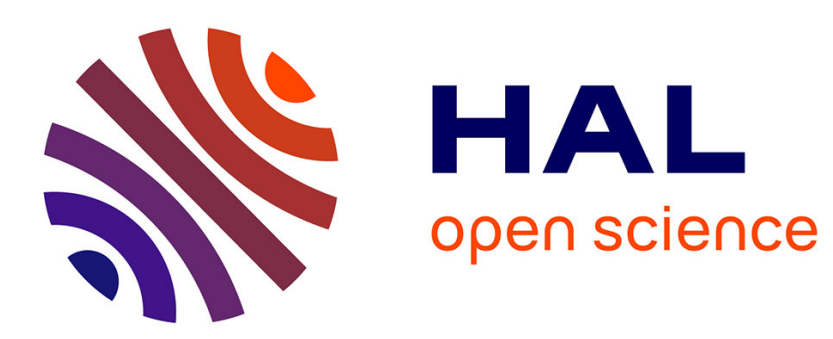

\title{
Le travail comme échange chez Saint-Exupéry : penser l'articulation des trois dimensions du travail
}

\author{
Bernard Guéry
}

\section{To cite this version:}

Bernard Guéry. Le travail comme échange chez Saint-Exupéry : penser l'articulation des trois dimensions du travail. Revue de Philosophie Economique / Review of Economic Philosophy, 2016, 17 (2), pp.83-123. 10.3917/rpec.172.0083 . hal-02508868

\section{HAL Id: hal-02508868 \\ https://hal.science/hal-02508868}

Submitted on 16 Mar 2020

HAL is a multi-disciplinary open access archive for the deposit and dissemination of scientific research documents, whether they are published or not. The documents may come from teaching and research institutions in France or abroad, or from public or private research centers.
L'archive ouverte pluridisciplinaire $\mathbf{H A L}$, est destinée au dépôt et à la diffusion de documents scientifiques de niveau recherche, publiés ou non, émanant des établissements d'enseignement et de recherche français ou étrangers, des laboratoires publics ou privés. 


\title{
Le travail comme échange chez Saint-Exupéry : penser l'articulation des trois dimensions du travail
}

\author{
Bernard GUERY ${ }^{1}$
}

\section{Résumé}

Les apports pour la gestion de la conception du travail de Saint-Exupéry dans Citadelle constituent l'objet de cet article. Après avoir présenté le "schème herméneutique " (Sabot, 2002), qui constitue le cadre méthodologique, le travail comme échange en gestion est brièvement présenté pour qu'apparaisse ensuite dans toute son originalité la vision du travail comme échange chez Saint-Exupéry. Il s'agit pour le travailleur d'échanger sa vie contre l'éternité, pour s'arracher aux prises du temps qui mène à la mort, en se transférant dans l'empire, via l'œuvre accomplie. Nous montrons comment, grâce à la pensée de SaintExupéry, une articulation organique des trois dimensions du travail du modèle de Gomez (2013) et envisageable. C'est, en effet, dans la réalisation d'une œuvre (objectif) contre un salaire qui permet de consommer, que le travailleur se donne plus profondément lui-même à l'empire (collectif), don qui le fait advenir à un être plus grand (subjectif). Cette articulation est alors propre à questionner trois éléments du contexte managérial : le lien entre le travail et la réussite de la vie, les difficultés des cycles courts de l'emploi, la prise en compte des parties prenantes.

Mots clés : Saint-Exupéry, travail, travailleur, gestion, échange

\begin{abstract}
The contributions of the conception of labor in Saint-Exupéry's Citadelle for the management constitute the object of this work. Having presented the "hermeneutic schema" (Sabot, 2002), which establishes the methodological frame, labor as exchange in management is briefly presented so that appears then in all its originality the vision of the work as exchange to Saint-Exupéry. The matter is for the worker to exchange its life against eternity, to tear away from the grips of the time that leads to death, by transferring in the empire, through the accomplished work. The paper shows how, thanks to Saint-Exupéry's thought, an articulation of the three dimensions of work is conceivable, from the Gomez (2013) model. By realizing a work for money to consume, the worker gives himself to the empire. This gift gives him back a better being. This articulation is appropriate to question three elements of the managerial context: the link between the work and the success of life, the difficulties of short employment cycles and the consideration of the stakeholders.
\end{abstract}

Keywords: Saint-Exupéry, work, worker, management, exchange

Classification JEL : A12

\footnotetext{
${ }^{1}$ Enseignant-Chercheur à l'IPC

Faculté libre de Philosophie et de Psychologie

bguery@ipc-paris.fr
} 


\section{INTRODUCTION}

Babeau et Chanlat qualifient les chercheurs en sciences de gestion de «contrebandiers du savoir» (2011, p. 34). L'expression suggère l'idée d'un passage illégal de savoir au-delà des frontières entre disciplines. En suivant cette idée, on peut considérer qu'il n'est pas a priori inopportun d'aller chercher en dehors de la gestion des apports féconds pour cette discipline.

De plus, la crise économique peut-être vue aussi comme une invitation à aller chercher dans d'autres domaines un sens qui permette d'éclairer les situations des entreprises au moment où les fondements de leur système sont remis en cause.

Par ailleurs, les réflexions sur le travail mettent parfois en lumière son caractère ambigu. Cugno souligne le sens paradoxal du travail, entre «aliénation et réalisation de soi » (2006, p. 59). Ce paradoxe n'est pas exclusif à notre époque. Pietr (2004) s'en fait le témoin en écrivant que le verbe « travailler » véhicule toujours des valeurs particulières chargées de douleurs, et aussi d'espoir. Quand le christianisme énonce : "Tu gagneras ton pain à la sueur de ton front », le travail est vu comme une malédiction, mais c'est aussi un instrument de rédemption puisque c'est par lui que nous nous sauverons. Pour le Marx, du premier volume du Capital, « c'est parce que le prolétaire est le produit le plus achevé de l'aliénation par le travail qu'il est la figure de l'homme nouveau [...] Cet homme nouveau transformera le travail, pris comme forme même de la servitude, en instrument de libération et d'épanouissement $\gg(2004$, p. 81-82).

Aujourd'hui, la souffrance au travail est, à bien des égards, une problématique qui se situe au cœur des préoccupations d'une partie de la recherche en gestion. Or, cette souffrance vient souvent de la perte de sens. Les hommes et les femmes dans l'entreprise ont de plus en plus de difficultés à trouver du sens à leur travail (Berthoin Antal et Frémeaux, 2013, p. 5). En effet, « avoir un emploi ne suffit pas. Il faut qu'il ait du sens... L'absurdité croissante qui caractérise nos organisations est ressentie d'autant plus douloureusement que les attentes à l'égard du travail restent fortes. » (Michel, 2012, p. $3)$.

C'est pourquoi nous proposons de solliciter la conception du travail d'Antoine de Saint-Exupéry, qui permet de manifester certains aspects du travail propre à lui donner du sens. La démarche employée consiste à se saisir d'une réalité mise en lumière par les sciences de gestion (ici les trois dimensions du travail) pour l'éclairer à partir d'un point de vue nouveau, et ainsi déceler des aspects inédits (ici le mode d'articulation des trois dimensions du travail et leurs implications pour la gestion).

L'idée-force que l'on peut tirer de l'œuvre de Saint-Exupéry pour la conception du travail regarde la relation du travail au sujet travaillant. Ce que l'on pourrait proposer comme hypothèse à vérifier selon les méthodes propres des la gestion, serait que le travail est moins fait qu'il ne fait celui qui le 
fait. Il faut ajouter à cela la précision suivante : ce que fait le travail chez celui qui le fait, c'est la permanence et l'inscription nodale dans la communauté. Cette conclusion est obtenue au moyen d'un renversement de perspectives stimulant pour la réflexion. En effet, l'œuvre devient l'ouvrier, le don de soi est d'abord un don à soi, par la médiation de la totalité communautaire et temporelle. Lire SaintExupéry constitue donc une invitation toute particulière, que nous voudrions expliciter, à reconsidérer l'aspect subjectif du travail, ainsi que son aspect collectif, en plus de l'aspect objectif, pour reprendre la terminologie de Gomez (2013). C'est surtout leur articulation qui devient pensable, puisque la production objective, chez Saint-Exupéry devient un vecteur de l'établissement de la collectivité de laquelle et dans laquelle le travailleur lui-même (subjectif) se trouve grandi.

Notre analyse de sa pensée sur le travail se fonde principalement sur le texte de Citadelle (SaintExupéry, 1948), émaillé de commentaires opportuns, notamment Ravoux (1980), Quesnel (1989), Kerneff (2001), Bollnow (1981). Néanmoins, nous privilégierons l'approche directe du texte. Pour montrer l'apport original de la pensée de Saint-Exupéry, nous ferons dialoguer certains thèmes qu'il développe avec des pensées de ses contemporains élaborées dans un registre discursif plus conceptuel, notamment Arendt et Sartre. C'est dans l'optique du schème interprétatif (Sabot, 2002) que nous opérerons la transposition sur la portée des sciences de gestion, c'est à dire que, contrairement à d'autres schèmes (Badiou, 1998) de relation de la poésie à la pensée, on considère que le texte de Saint-Exupéry exprime des idées sur un mode poétique, et que l'interprétation consiste à exprimer sur un mode conceptuel.

Pour manifester l'originalité de la pensée de Saint-Exupéry sur la question, on peut, après avoir défini méthodologiquement l'usage que nous ferons de ses textes, mettre sa conception du travail, qu'il définira comme un «échange », en regard d'autres conceptions du travail qui lui donnent aussi cette caractéristique. Cette conception rend possible l'articulation des dimensions du travail qui s'expriment concrètement dans trois apports principaux pour la gestion.

\section{LE CADRE INTERPRETATIF : LE SCHEME DIDACTIQUE}

Il peut paraitre étonnant de solliciter la pensée d'un homme de lettres pour éclairer une réalité comme le travail. En effet, cette réalité est habituellement étudiée dans des disciplines telles que la gestion, la sociologie, ou l'économie.

On peut pourtant prendre acte de précédents comme le travail de Noël et Le Loarne (2007), qui sollicitent la pensée de Simone Weil pour éclairer la réalité du travail et de l'organisation responsable. On peut mentionner également la journée d'étude de l'ISG sur «Hannah Arendt, Les Sciences de Gestion et le Management»( 2 octobre 2013). Leclercq-Vandelanoitte mène une démarche de cet ordre avec Foucault (2011) et Leclercq-Vandelanoitte et Isaac avec Deleuze (2013). 
Mais il s'agit, dans les exemples cités, de philosophes. Au contraire, Saint-Exupéry est davantage étudié en littérature qu'en philosophie. C'est pourquoi il est important, avant d'importer en gestion la pensée de Saint-Exupéry, de savoir à partir de quelle discipline l'importation dont nous parlons va avoir lieu.

La question de la discipline à laquelle appartient Citadelle continue d'interroger (Bollnow, 1981, p. 41). Même si certains considèrent Saint-Exupéry comme un philosophe, comme le fait Ravoux «nous considérons la pensée de Saint-Exupéry [...] comme appartenant à celle que depuis si longtemps on recherche et dont la recherche s'appelle philosophie. » (1980, p. 106), on ne peut nier néanmoins l'aspect littéraire de la présentation de sa pensée.

Cet aspect littéraire, pour être audible en science de gestion, nécessite une traduction qui ne peut se faire sans précautions méthodologiques. Pour mettre en place cette méthodologie de traduction de façon sérieuse, il est précieux de solliciter la pensée de Badiou développée dans son Petit manuel d'inesthétique (1998). L'auteur cherche à manifester les différents types de relation de l'art à la pensée, ce qui est utile pour penser la relation d'un écrit littéraire abordant la question du travail à une réflexion académique en sciences de gestion. Au début de son ouvrage, l'auteur prend acte de «l'oscillation » (1998, p. 9) qui caractérise la relation de l'art à la pensée. Il écrit que « le maître philosophe demeure divisé, au regard de l'art, entre idolâtrie et censure. » (1998, p. 10). L'attitude du chercheur en gestion vis-à-vis d'un texte de Saint-Exupéry sur le travail peut également osciller entre ces deux attitudes : le rejeter au non de sa forme non-académique comme non pertinent, ou bien le prendre tel quel, sans transposition. Pour éviter ces deux écueils et penser de façon structurée une relation des sciences de gestion au texte de Saint-Exupéry, il faut recourir à trois schèmes possibles exposés par Badiou : le schème didactique, le schème romantique et le schème classique. Ces schèmes forment un cadre duquel la réflexion de Sabot invite à s'échapper pour trouver une forme de relation qui satisfasse les exigences du présent travail.

Selon le schème didactique, «l'art est incapable de vérité, et toute vérité lui est extérieure » (1998, p. 10). La pensée devra donc se servir de l'art de façon instrumentale, comme illustration par exemple, ou à des fins éducatives. Selon le schème romantique, au contraire, «l'art seul est capable de vérité. » (1998, p. 12, souligné dans le texte). Il accomplit alors ce que la philosophie ne peut qu'indiquer. Le schème classique, quant à lui, dénie à l'œuvre d'art toute valeur dans le champ de la connaissance.

Ces schèmes mèneraient le chercheur en gestion à manquer l'apport précieux du texte de SaintExupéry, ou à négliger de l'interpréter. Sabot (2002) propose un schème supplémentaire, dans le champ de la littérature, auquel il restreint la problématique de Badiou, et auquel appartient l'œuvre de Saint-Exupéry, qu'il appelle herméneutique (2002, p. 54). Dans ce schème, l'œuvre littéraire contient une vérité, et le penseur est amené à « rappeler cette vérité à l'ordre du concept, selon un process 
interprétatif qui opère en quelque sorte la traduction du langage littéraire, où cette vérité est inapparente, comme dissimulée à elle-même par son mode propre de manifestation, dans le langage philosophique, où elle reçoit son élucidation spéculative » (2002, p. 54, souligné dans le texte).

C'est ce schème herméneutique qui constitue le cadre théorique de notre travail. Ce schème herméneutique trouve une application particulièrement adaptée dans le cas du texte de Citadelle, dont la forme invite à l'utilisation de ce schème. En effet, si Saint-Exupéry parle en «paraboles » (note des éditeurs, Saint-Exupéry, 1948, p. 7), sans pour autant nous donner la clef, cela peut être vu comme une invitation à tirer nous-même la «morale » de la parabole, c'est-à-dire à interpréter son texte. Bien des critiques, pourtant, on eu des difficultés à interpréter le texte (Quesnel, 1989, p. 63).

En effet, et ce que nous allons dire permet autant de manifester la tournure parabolique de Citadelle que d'introduire rapidement à l'ambiance de l'ouvrage, le livre de Saint-Exupéry se présente comme un soliloque méditatif du fils d'un seigneur berbère, à la structure libre, déliée, redondante à la manière d'une mélopée orientale ou d'un chant de la nature, aux formulations parfois sibyllines qui entourent les petits chapitres d'une atmosphère ésotérique envoûtante, où la violence se fait poésie, où la langueur s'épanouit au soleil, en contraste avec le mystère enfoui en chaque chose, comme dans une aurore animiste. Par-dessus tout, «tout est symbole» (Quesnel, 1989, p. 63), et «la parole [est] épaisse, faite de multiples strates de signification, cette parole dans laquelle un chat est toujours autre chose qu'un chat tout en restant un chat. » (Ibid.)

Ce dernier caractère du texte de Saint-Exupéry le dégage de soi des essais de conceptualisation rapides. Le lecteur est devant une pensée qui se cherche en abordant ses thèmes, qui se répète en s'amplifiant d'autres thèmes, qui ne laisse rien derrière elle, au sens où tout ce qui précède un passage est présent dans ce passage, charrié par de multiple évocations qui sont autant de renvois formant un réseau inextricable. Vouloir analyser, au sens premier, c'est à dire résoudre en ses éléments primitifs un tel réseau, c'est en perdre le charme, et avec lui la vérité. Et l'on peut dire avec Saint-Exupéry :

« Ainsi de ceux qui croient le découvrir en le divisant, mon territoire. "Il y a là, disentils, des moutons, des chèvres, de l'orge, des demeures et des montagnes - et quoi de plus ?" Et ils sont pauvres de ne rien posséder de plus. Et ils ont froid. Et j'ai découvert qu'ils ressemblent à celui-là qui dépèce un cadavre. "La vie, dit-il, je la montre au grand jour : ce n'est que mélange d'os, de sang, de muscles et de viscères.” » (Saint-Exupéry, 1948, p. 29).

Néanmoins nous allons essayer, en nous empêchant de donner un caractère définitif et systématique à la pensée que nous présentons, d'en tirer selon le schème herméneutique, quelques grandes lignes riches d'enseignements pour la gestion. 


\section{TRAVAIL ET ECHANGE EN GESTION}

Saint-Exupéry conçoit le travail comme un échange, nous aurons l'occasion de le montrer. Pour que cette conception apparaisse dans son originalité, il est pertinent d'évoquer d'abord brièvement d'autres conceptions du travail comme échange, pour donner un élément de comparaison.

\subsection{Le travail comme marchandise}

La notion de travail est ambivalente et nombreuses sont les significations qu'on lui attribue :

« Nous disposerions aujourd'hui d'un concept de travail constitué de plusieurs couches de signification, dont chacune est susceptible de constituer une dimension du travail actuel, notamment une des dimensions subjectives du rapport au travail : le travail, pour les individus, est en effet à la fois conçu comme un effort, une activité fatigante de participation à la production, en contrepartie de laquelle ils obtiennent un revenu. Mais le travail est également vécu comme une activité qui doit permettre aux individus de s'exprimer. Enfin, il est apprécié pour les droits sociaux auxquels il ouvre, en tant qu'emploi. Les enquêtes sociologiques dont nous disposons confirment la coexistence de cette pluralité de sens » (Méda, 2010, p. 57).

Pour une comparaison féconde avec la pensée de Saint-Exupéry, il faut évoquer celles qui sont les plus proches de celle décrite dans Citadelle. Il s'agit des conceptions qui placent le travail dans le voisinage de la notion d'échange, donc du point de vue économique et juridique. Dans ces disciplines, le travail est conçu parfois comme une marchandise à échanger. C'est le cas de la vision de l'école néo-classique : «s'est progressivement construite, au sein du courant libéral — ou, plus précisément, à travers l'école classique anglaise et l'école néo-classique - la représentation du travail comme simple marchandise » (Maréchal, 2001, p. 457). Cette conception du travail comme marchandise à échanger le rend extérieur à l'homme (de l'ordre de l'avoir et non de l'être, pourrait-on dire pour simplifier), indépendant de son être. La conception marxiste fait du travail abstrait une mesure dans l'échange pour former la valeur des marchandises : «tout travail est [...] dépense, dans le sens physiologique, de force humaine et à ce titre de travail humain égal, il forme la valeur des marchandises » (Marx, 1985, p. 61).

\subsection{Le travail comme échange}

Certaines conceptions du travail ne le perçoivent pas comme une marchandise à échanger, ni comme une valeur de la marchandise, mais comme un échange. Elles se trouvent davantage dans des postures issues de la sociologie.

L'échange peut se définir ainsi : «mutual giving and receiving of both material and non material things » (Bowman, 1965, p. 247-248). Il y a différents types d'échange, parmi lesquels l'échange de 
dons (Davis, 1996, p. 274). Les conceptions du travail comme échange se développent notamment dans le cadre de la théorie du don, issue de l'Essai sur le don de Mauss (2012), qui interprète dans les sociétés archaïques comme un échange de don ce que l'on prenait auparavant trop facilement pour du troc (Cazeneuve, 1968, p. 99). Ainsi de Akerlof $(1982,1984)$, qui conçoit le travail comme un échange de cadeaux (cf. Godbout, 1992, p. 117), ou encore Alter qui constate que les entreprises cherchent à éviter de voir comme un don l'investissement des salariés, pour ne pas avoir à le célébrer (2010, p. 193). C'est davantage à partir de la sociologie et de l'anthropologie que se construisent ces représentations du travail (Davis, 1996, p. 274). Mais on sollicite aussi cette conception du travail comme échange en éthique des affaires : "Le deuxième principe de justice est le mérite. Dans la mesure où le travail est un échange entre un effort, un coût, et une rétribution, l'équité de cet échange, généralement mesurée sur des comparaisons continues, fixe la juste sanction du mérite. Quand le déséquilibre est trop grand entre les contributions du salarié et ses rétributions, le principe de mérite peut laisser la place à un sentiment d'exploitation »(Dubet, 2005, p. 11).

Quoiqu'il en soit, dans toutes les conceptions du travail comme échange, et non comme marchandise, le travail, en entrant dans la catégorie échange, met en place quatre termes. Les deux personnes qui échangent, et les deux choses échangées. Le salarié et l'entreprise s'échangent des biens. Le premier fournit un «effort» (Dubet, 2005, p. 11) au sens large, ou une compétence, un engagement personnel, des ressources sociales, et le second lui fournit un salaire, une reconnaissance, un statut social, etc.

\section{LE TRAVAIL COMME ECHANGE DANS CITADELLE DE SAINT- EXUPERY}

La conception de Saint-Exupéry du travail comme échange est spécifiquement différente. Pour la comprendre, il faut la replacer dans son contexte, celui du sens de la vie pour Saint-Exupéry.

\subsection{Le but : durer}

Le travail est, pour Saint-Exupéry, un moyen de conjurer le sort de la mortalité inhérente à l'homme. L'homme désire l'éternité, à l'image de son écrin, la nature, qui cherche sa stabilité :

Il est un temps pour la création, mais il est un temps pour la créature. Il est un temps pour la foudre écarlate qui rompt les digues dans le ciel, mais il est un temps pour les citernes où les eaux rompues vont se réunir. Il est un temps pour la conquête, mais vient le temps de la stabilité des empires : moi qui suis serviteur de Dieu, j'ai le goût de l'éternité. Je hais ce qui change » (Saint-Exupéry, 1948, p. 27). 
Le corollaire de cette haine du changement, c'est le désir de la pérennité. En parlant de l'homme : «Le seul intérêt qui le meuve n'est que celui d'être permanent et de durer. » (Saint-Exupéry, 1948, p. 215). Un texte tiré des Carnets fait écho admirablement à ce désir d'éternité exprimé dans Citadelle: «Rendez-nous, disent avant tout les hommes, rendez-nous "l'éternité" [...] rendez-nous cela que nous sommes et qui dure au-delà de nous-mêmes. » (1994, p. 511).

Il s'agit donc de durer. Mais il y a deux types de durée pour Saint-Exupéry: «je distingue la continuité de la routine, la stabilité de la mort » (Saint-Exupéry, 1948, p. 114). L'éternité est, en effet, un terme polysémique dans l'œuvre de Saint-Exupéry (Kerneff, 2001, p. 83). Celle qu'il faut atteindre est la continuité, la stabilité qui est bâtisseuse : «il est bon que le temps qui s'écoule ne nous paraisse point nous user et nous perdre, comme la poignée de sable, mais nous accomplir. Il est bon que le temps soit une construction » (Saint-Exupéry, 1948, p. 29).

Par conséquent, puisque durer est l'enjeu fondamental, «la mort est bien le problème originel » (Ravoux, 1980, p. 108). Il s'agit d'y échapper d'une façon où d'une autre. Et l'on peut dire que la question la plus grave est celle qui est posée au jeune chef berbère : « où irais-je une fois mort?».

Toutefois, cette question est moins celle d'une vie après la mort dans un au-delà, que dans ce monde. En effet, c'est un soldat éloigné de ses fils qui parle ainsi, et qui dit : «J'ai des fils [...] et ils grandissent, et je ne les aurai pas enseigné. Je ne dépose donc rien en eux. Et où irais-je une fois mort ?» L'enjeu est donc de laisser quelque chose après soi, ici un enseignement dans ses fils.

La solution pour résoudre ce problème de la mort serait donc de passer dans quelque chose qui dure après soi, comme les enfants pour le guerrier.

\subsection{Le moyen : s'échanger}

Le travail est, pour Saint-Exupéry, ce moyen d'échapper à la mort et d'atteindre l'éternité en quelque sorte. Un paragraphe permet de voir émerger l'idée que nous voulons présenter :

Et je m'en fus parmi mon peuple songeant à l'échange qui n'est plus possible lorsque rien de stable ne dure à travers les générations, et au temps qui coule alors, inutile, comme un sablier. Et je songeais : cette demeure n'est point assez vaste et l'œuvre contre laquelle il s'échange n'est point assez durable encore. Et je songeais aux pharaons qui se firent bâtir de grands mausolées indestructibles et anguleux et qui avancent dans l'océan du temps qui les use lentement en poussière [...]. Et je songeais : il n'est point assez durable, ce temple avec sa charge de dorures et d'objets précieux qui ont coûté de longues vies humaines, avec ce miel enfermé de tant de générations, avec ces filigranes d'or, ces dorures sacerdotales contre lesquelles de vieux artisans se sont lentement échangés et ces nappes brodées sur lesquelles 
des vieilles tout au long de leur vie se sont lentement brûlé les yeux, et, une fois racornies, toussotantes, ébranlées déjà par la mort, ont laissé d'elles cette traîne royale. Cette prairie qui se déroule. Et ceux qui l'aperçoivent aujourd'hui se disent : “Qu'elle est belle, cette broderie ! Qu'elle est donc belle...” Et je découvre que ces vieilles ont filé leur soie dans leur métamorphose. Ne se sachant point aussi merveilleuses » (Saint-Exupéry, 1948, p. 42).

On y voit apparaître l'idée de l'œuvre qui dure après soi et dans laquelle l'ouvrier se coule, se transfère pour échapper à la mort. Saint-Exupéry décrit précisément ce transfert en parlant d'un vieil artisan :

Je méditais devant l'un d'entre eux qui était aveugle et qui avait de plus perdu sa jambe. Si vieux, si moribond, tout geignant comme un vieux meuble chaque fois qu'il se remuait et qui répondait lentement car il était très vieux en âge et perdait la clarté des mots, mais qui devenait de plus en plus lumineux et clair et compréhensif dans l'objet même de son échange. Car de ses mains tremblantes il ajoutait encore son travail devenu élixir de plus en plus subtil. Et lui, s'évadant si merveilleusement de sa vieille chair racornie, devenait de plus en plus heureux, de plus en plus inattaquable. De plus en plus impérissable. Et, mourant, ne le savait point, les mains pleines d'étoiles... » (Saint-Exupéry, 1948, p. 43).

En travaillant à son tour, l'artisan s'est échangé contre son œuvre, œuvre qui dure après lui et qui le transporte par conséquent, au-delà de la mort : «Et j’ai appris ceci qui est essentiel : à savoir qu'il importe de bâtir d'abord le navire et de harnacher la caravane et de construire le temple qui dure plus que l'homme. Et désormais les voilà qui s'échangent dans la joie contre plus précieux qu'euxmêmes. » (Saint-Exupéry, 1948, p. 43). Ce contre quoi ils s'échangent est plus précieux qu'euxmêmes parce que cela dure, et il n'y a de valeur que de ce qui dure. Dans le travail, on a donc un processus de recréation dans l'objet (Saint-Exupéry, 1948, p. 45).

Cette idée de la pérennité de l'homme qui laisse quelque chose de lui-même après lui n'est pas nouvelle. Toutefois, c'est habituellement à la génération que l'on prête cette fonction. Pour Aristote, par exemple, qui parle de la procréation, « comme dans les autres espèces animales et chez les plantes, il s'agit d'une tendance naturelle à laisser après soi un autre être semblable à soi » (2014, p. 25). On peut donc dire que Saint-Exupéry reprend l'idée classique de la pérennité de l'homme dans la génération, qui se reproduit par une tendance naturelle à laisser quelque chose après lui, pour se survivre à lui-même. Mais l'auteur de Citadelle transfère cette idée de l'ordre de la nature à l'ordre du faire, de l'ordre de la procréation à celui de la création, de telle sorte que produire serait, en quelque sorte, engendrer. C'est pourquoi la production, le travail, donne sens à la vie : « la vie n'a de sens que si l'on s'échange peu à peu », (Saint-Exupéry, 1948, p. 41) et par conséquent, «ceux là qui n'échangent rien ne deviennent rien » (Saint-Exupéry, 1948, p. 45). 
La conséquence de cette conception est qu'il est absurde de travailler pour gagner sa vie. En effet, travailler, c'est perdre sa vie contre autre chose, pour gagner l'éternité. Si l'on travaille pour gagner sa vie, et non l'éternité, on joue à perte : «N'espère rien de l'homme qui travaille pour sa propre vie, et non pour son éternité $[\ldots]$. S'ils se bâtissent des maisons pour y vivre, à quoi bon échanger leur vie contre leur maison ? Puisque cette maison doit servir leur vie et rien d'autre » (Saint-Exupéry, 1948, p. 43). De ce point de vue, une analogie avec la pensée de Arendt sur l'improductivité du travail par rapport à l'œuvre sera féconde, nous le montrerons dans la suite. En effet, pour elle, «tout ce que produit le travail est fait pour être absorbé presque immédiatement dans le processus vital» (2014, p. 145).

Mais avec qui fait-on l'échange ? Comme dans tout échange, en effet, il est nécessaire qu'il y ait quatre termes. Pour Ravoux, il s'agit d'un dieu : «pour l'échange, il faut un Dieu, comme le domaine ou le temple, le poème ou la communauté, qui reçoit la part que l'homme délègue et en laquelle il s'échange.» Cette mention n'est pas présente en tant que telle dans le texte de Saint-Exupéry. En revanche, le texte de Citadelle porte une phrase suggestive à ce sujet: "c'est dans le temps que baignent d'abord, pour devenir le fruit, la broderie ou la fleur » (1980, p. 44-45). Le temps possède ce qui est échangé contre la vie. La brodeuse donne sa vie au temps en faisant sortir la matière de la broderie qui était dans le temps, et la faire passer, et soi avec elle, dans l'éternité. Il s'agirait donc de prendre au temps l'éternité, et de lui donner en échange sa vie. On se situe donc à l'opposé d'une autre conception du travail pour laquelle, « son essence, c'est le temps » (Méda, 2010, p 15). Méda se réfère à la conception smithienne, que l'on retrouvera sous la plume de Marx avec l'idée de «travail abstrait », « le travail est précisément avant tout une unité de mesure, un cadre d'homogénéisation des efforts, un instrument permettant de rendre les différentes marchandises comparables. Son essence, c'est le temps. » Pour expliquer cette phrase de Méda, il serait nécessaire de faire un détour par la définition du temps comme «mesure » ou «nombre » du mouvement, issue d'Aristote (2002, p. 252). Quoiqu'il en soit, chez Saint-Exupéry, le temps n'est pas le travail, mais ce avec quoi, l'on s'échange.

Si l'on s'en tenait là, il semble qu'un manque dans ce que nous avons reconstitué de la pensée de Saint-Exupéry se fasse jour. En effet, tout ce que l'on produit ne dure pas plus celui qui le produit, comme dans cette belle image : «Et je passai devant mon savetier à la jambe unique occupé d'embellir de filigranes d'or ses babouches et je compris bien, malgré qu'il n'eût plus de voix, qu'il chantait : "Qu'y a-t-il, savetier, qui te rend si joyeux ?" Mais je n'écoutai point la réponse, sachant qu'il se tromperait et me parlerait de l'argent gagné ou du repas qui l'attendait ou du repos. Ne sachant point que son bonheur était de se transfigurer en babouches d'or " (Saint-Exupéry, 1948, p. 48). Or, les babouches d'or ne sont pas éternelles. Comment, dès lors, expliquer que le travail soit vecteur d'éternité si ce qu'il produit ne dure pas éternellement? 
Il semble que pour comprendre le lien entre le travail, fût-il celui du savetier, et la possibilité de toucher l'éternité, il faille passer par la médiation de ce qui est dénommé par Saint-Exupéry l'empire. L'image de l'empire est omniprésente dans Citadelle. De ce terme suggestif il est difficile de tirer une circonscription conceptuelle claire. L'empire pourrait éventuellement se définir comme le tout, plus grand que la somme de ses parties, qui contient les hommes et leur production en lui. Mais il ne désigne pas seulement la communauté des hommes existant à un instant donné, organiquement structurée. L'empire est le nœud qui relie le présent, le passé et le futur de la communauté. Or, c'est par la médiation de l'empire que le travail peut procurer au travailleur l'éternité.

En réalité, c'est l'empire contenant les savates qui dure plus que l'homme, et en passant dans ses savates, le savetier passe en fait dans l'empire qui dure après lui « et si je vous convie de collaborer et d'être ensemble et de constituer une grand figure qui enrichisse chacun, qui participe de tous, et l'enfant de l'empire [...], comment n'en seriez-vous pas augmentés et comment résisteriez-vous ?» (Saint-Exupéry, 1948, p. 72). Ainsi l'homme est à la fois bâtisseur de l'empire et pierre de cet édifice qui l'emporte avec lui après sa mort. Ainsi l'ensemble des hommes, la vielle femme par sa broderie, le savetier par sa cordonnerie, et chacun à son niveau, construisent l'empire et l'arrachent aux prises du temps. Derrière cette métaphore, on peut lire que la contribution de chacun, par son travail, à la société, laisse un peu de lui dans cette société qui dure alors après lui et l'emporte avec elle.

\subsection{Le don de soi comme modalité de cet échange : le travail à double-fond}

L'échange peut bien sûr être conçu comme marchand, mais il peut aussi se revêtir d'une modalité de don. Le titre lui-même de l'ouvrage de Mauss s'en fait l'écho : Essai sur le don. Forme et raison de l'échange dans les sociétés archaïques (2012). Le don est placé d'emblée dans la perspective possible de l'échange. Pour Darmangeat, «un don peut éventuellement, lui aussi, être accompagné d'un transfert en sens inverse (un contre-don), mais celui-ci n'est jamais exigible : si le récipiendaire peut se sentir tenu de le fournir, cette obligation reste purement morale ; son non-respect peut entraîner une réprobation, mais nul emploi légitime de la force sociale» (Darmingeat, 2016, p. 23). C'est donc l'exigibilité qui différencie les deux formes d'échange. On peut donc se poser la question : l'échange que constitue le travail chez Saint-Exupéry se caractérise-t-il par une exigence de retour? Autrement dit, le travailleur achète-t-il son éternité ?

En réalité, dans les textes de Citadelle, les deux conceptions semblent se superposer. Car l'échange se fait à deux niveaux, l'un explicite et banal, le second enfoui et plus réel. Nous avons déjà cité ce texte plus haut, dans lequel les deux niveaux apparaissent : «je n'écoutai point la réponse, sachant qu'il se tromperait et me parlerait de l'argent gagné ou du repas qui l'attendait ou du repos. Ne sachant point que son bonheur était de se transfigurer en babouches d'or. » (Saint-Exupéry, 1948, p. 48). Le salaire est exigé, mais pour exiger le bonheur, encore faut-il le verbaliser, en être conscient, ce qui n'est pas le cas du travailleur ici. 
Par conséquent, il ne s'agit pas de nier l'échange marchand, mais ce dernier apparaît comme un véhicule superficiel, qui permet, supporte, voile et protège un échange plus profond, non plus entre un effort et un salaire, mais entre le travailleur et son destin. Ce point nous semble être l'aspect le plus éclairant de la pensée de Saint-Exupéry sur le travail. Ce dernier constitue un double échange. Le premier, échange marchand, visible « pour les yeux » (Saint-Exupéry, 1946, p. 72), est fourniture d'un effort en échange d'un pouvoir de consommer. Le second, au sein du premier, visible « avec le cœur» (Saint-Exupéry, 1946, p. 72), est l'échange de dons entre le don de soi du travailleur et l'éternité que lui fournit son inscription dans l'empire grâce à la réalisation superficielle de l'objet.

On peut apercevoir deux conséquences de ce phénomène du travail à double fond. La première réside dans le réalisme de l'économie du don. Cette dernière fait l'objet, de la part du courant néolibéral, du respect que l'on veut bien consentir aux utopistes et aux idéalistes. Mais les penseurs du courant économique néo-libéral se targuent de voir, eux, la réalité, et, les concernant, le pragmatisme serait parfois un euphémisme pour désigner le cynisme. Forts de ce présupposé, ils évacuent le don de leurs objets de recherche, ou, plus subtils, ils le lisent à la lumière de leurs présupposés, comme quand ils expliquent l'altruisme par une maximisation de l'auto-réputation (Tirole, 2009, p. 578). Comme le dit Gomez, « le mystère du don, c'est que depuis plus de deux siècles, c'est à dire depuis l'avènement de l'économie moderne, sa place a été réduite dans la préoccupation des économistes "sérieux" qui ne se sont consacrés qu'à comprendre les échanges marchands, les calculs et les intérêts, et ont amputé leur science d'une partie de son objet» (2015, pp. 27-28). En réalité, il ne s'agit pas de dire qu'il faudrait plus de don en économie, mais, qu'il faut changer de paradigme pour voir le don là où il existe, c'est à dire partout là où existe l'échange marchand (2015, p. 39), qui commencerait toujours par un don, celui de la confiance. La pensée de Saint-Exupéry trouve un écho dans ces théories dans la mesure où elles ne prônent pas davantage de don à côté de l'échange marchand, mais où elles voient du don au cœur même de l'échange marchand. Saint-Exupéry n'invite pas à remplacer le travail par le don, mais à voir que le travail, au delà de l'échange marchand, est aussi un don.

La seconde conséquence de ce phénomène de «double fond» du travail, est l'alternative entre deux attitudes vis-à-vis du travail, en fonction des deux regards que l'on porte sur la partie émergée de l'échange (effort contre salaire, et son corollaire, la consommation). On peut en effet, voir ce cycle travail-consommation comme une « icône » ou bien comme une «idole » : la face émergée du travail voile ou dévoile la partie immergée. Cette dialectique du caché et du dévoilé, que l'on retrouve dans la célèbre formule « on ne voit bien qu'avec le cœur. L'essentiel est invisible pour les yeux » (SaintExupéry, 1946, p. 72) va engendrer deux attitudes possibles face à l'échange effort-salaire et à la consommation que constitue le travail. On peut s'y arrêter, s'en tenir au regard des yeux, comme sur une idole qui arrête à elle-même l'intention: «Tu crois que ta joie viens de recevoir et d'acheter, comment te souviendrais-tu du contraire, quand on a fait tellement d'efforts pour te créer des liens avec l'objet ?» (p. 201). Alors, l'assoupissement mortifère guette : « les provisions sont nécessaires, mais plus dangereuses que la famine» $(1948$, p. 56). 
Mais on peut aussi le voir comme le signifiant d'un signifié plus profond. L'objet fabriqué ou consommé n'est alors qu'un vecteur, une icône, qui ouvre le regard sur une réalité plus haute. On ne voit bien qu'avec le cœur. Dès lors, s'arrêter à l'objet, c'est prendre le moyen pour la fin.

Il est nécessaire de présenter une différence fondamentale entre les deux niveaux d'échange en question. Le premier niveau d'échange, d'ordre marchand, est non-simultané et non identique. Ce qui est fourni (un effort) est différent de ce qui est reçu (un salaire), et les deux actes sont distincts. Il n'en va pas de même pour le niveau d'échange plus profond (don de soi contre éternité). Un passage énigmatique de Citadelle le manifeste : «Je te le dis, la grande erreur est d'ignorer que recevoir est bien autre chose qu'accepter. Recevoir est d'abord un don, celui-de soi-même. Avare non pas celui qui ne se ruine pas en présents, mais celui qui ne donne point la lumière de son propre visage en échange de ton offrande » (p. 206). Ce passage introduit une spécificité de l'échange immergé dont il est question dans le travail. C'est ici que la conception de Mauss doit être abandonnée, bien qu'elle serve de berceau conceptuel à l'élaboration des échanges de don. En effet, dans l'échange de don que Mauss décrit des sociétés primitives, le don est autre chose que le contre-don. Une autre chose fait l'objet du contre-don, que ce soit simultanément (on peut prendre l'exemple des cadeaux de Noël dans notre société) ou plus tard (l'invitation à dîner que l'on se rend). Chez Saint Exupéry, il ne s'agit pas d'une autre chose. Le passage cité plus haut le montre : Recevoir, c'est donner. Par conséquent, donner c'est recevoir, comme le dit l'auteur plus loin : «Car ce que tu donnes en réalité ne te diminue point, mais bien au contraire t'augmente dans tes richesses à distribuer» (p. 181) ; «Quand tu donnes, tu reçois plus que tu ne donnes» (p. 349). Plus spécifiquement vis-à-vis du travail, il écrit: "Vous ne connaîtrez point la jubilation de l'homme qui livre son œuvre, laquelle ne lui sera point rendue. [...] Je te demande de vivre non de ce que tu reçois, mais de ce que tu donnes, car cela seul t'augmente » (p. 199). Ici, l'échange n'est pas la somme de deux actes distincts réciproques, mais un seul acte qui a valeur de don et de contre-don. On conçoit aisément que si on parle d'échange ici, c'est de façon analogique par rapport à l'échange de premier niveau (effort/salaire).

\subsection{Comparaison avec le don chez Sartre}

Cette notion de don articulé à une démarche existentielle n'est pas l'apanage de Saint Exupéry à cette époque. Pour prendre un exemple, on peut faire lien avec une pensée contemporaine, celle de Sartre, qui développe le concept de générosité. Ce faisant on montrera la spécificité de la valeur du don chez Saint-Exupéry, qui ne se comprend qu'en regard d'une réception.

On comprend d'emblée pourquoi la générosité peut avoir une place prépondérante dans une pensée qui met au premier plan une liberté non finalisée qui aura pour signe la gratuité. Pour Sartre, la générosité est « l'image de la liberté, comme Descartes l'a vu (p. 639). Dans les écrits de Sartre, la elle s'épanouit sur trois registres: esthétique, morale, et anthropologique. D'abord, dans le registre esthétique, elle est la caractéristique de toute écriture. En effet, l'écriture est l'offrande au lecteur 
d'une « liberté finie apprivoisant l'opacité du monde» (Louette, 2002, p. 72), le don d'un monde recréé. L'écriture est alors moins une vision descriptive, mais une engagement, un action. L'auteur de Qu'est ce que la littérature? en appelle à une écriture de la praxis, qui ne s'en tient plus à la description. «Ce qui est particulièrement remarquable pour nous est que Sartre reconnaît justement en l'auteur de Terre des hommes un précurseur de cette littérature de la praxis, ce qui montre bien la grande cohérence qui existe chez Saint-Exupéry entre ses idées théoriques et son propre travail d'écrivain » (Ambert, 2001, p. 105).

Au-delà d'une définition de l'écriture, la générosité va se déployer, chez Sartre, dans le registre éthique, et par conséquent (comment pourrait-il en être autrement chez Sartre ?) anthropologique : « la morale ici ne peut que [...] nous amener par l'aide de la réflexion non-complice à poser comme thème explicite de notre conduite ce qui était son sens implicite: la générosité absolue et sans limites, comme passion proprement dite et comme seul moyen d'être. » (1983, p. 137). Le don fait être le donateur. Cette idée d'un don qui est créateur du donateur se retrouve dans Citadelle (pp. 58, 158, 181-182, 199).

La différence entre les deux auteurs, qui empêcherait de classer Saint Exupéry dans le giron de l'existentialisme, si l'on en était tenté, tient au corollaire du don : la réception. Cette notion trouve peu d'écho dans l'œuvre de Sartre, et pour cause. Recevoir c'est accepter d'être débiteur, de se recevoir d'autrui. Dès lors on découvre une face plus sombre du don, coupé de la valeur morale de la réception : «La relation entre celui qui fait preuve de générosité et celui qui en profite est une relation qui instaure une certaine forme d'asservissement. Le bénéficiaire de l'acte généreux ce sont l'obligé du donateur» (Salzmann, 2000, p. 178). La générosité devient alors la «vertu cardinale du propriétaire » (Ibid.). Chez Saint-Exupéry, au contraire, recevoir est un acte profondément éthique, qui donne sens au don. On n'est donc pas devant un égoïsme larvé du donateur solipsiste qui agit pour se sentir exister, mais dans une authentique relation, qui a besoin de l'autre pour exister : «car pour donner à la courtisane il faudrait être plus riche qu'un roi, car ce que tu lui apportes, elles s'en remercie elle-même d'abord, et se flatte de sa réussite est s'honore soi-même d'être aussi habile et si belle qu'elle ait tiré de toi cette rançon. Et, dans ce puits sans fond, tu peux verser le chargement de mille caravanes d'or sans avoir commencé de donner. Car il faut quelqu'un pour recevoir» (1948, p. 205). Le don est donc le vecteur nécessaire de l'entrée en relation. Et cette relation permet à l'homme d'exister : l'homme se reçoit de la communauté, et ne peut être grand que dans la mesure où il est débiteur.

\section{IMPLICATIONS : TRAVAIL MORTIFERE DE L'HOMO FABER UNIFIE DANS}

\section{L'EMPIRE}

Cette conception du travail comme échange est lourde d'implications diverses qui peuvent sembler déroutantes. 
Tout d'abord, le travail n'est absolument pas conçu comme directement épanouissant pour l'homme. Il est proprement mortifère, du moins directement. En évoquant l'empire, Saint-Exupéry écrit :

Car on ne meurt point pour des moutons, ni pour des chèvres ni pour des demeures ni pour des montagnes. Car les objets subsistent sans que rien leur soit sacrifié. Mais on meurt pour sauver l'invisible nœud qui les noue et les change en domaine, en empire, en visage reconnaissable et familier. Contre cette unité l'on s'échange car on la bâtit aussi quand on meurt. La mort paie à cause de l'amour. Et celui-là qui eût lentement échangé sa vie contre l'ouvrage fait et qui dure plus que la vie, contre le temple qui fait son chemin dans les siècles, celui-là accepte aussi de mourir si ses yeux savent dégager le palais du disparate des matériaux, et s'il est ébloui par sa magnificence et désire s'y fondre. Car il est reçu par plus grand que lui et il se donne à son amour (Saint-Exupéry, 1948, p. 75).

Mais l'on voit par là-même que la mort est, dans ce sens, un accomplissement, comme si l'homme était un «être pour la mort» (Heidegger, 1986, p. 298). Le travail est destructeur de soi et reconstructeur de soi en dehors de soi, dans un tout plus grand que soi.

Une deuxième caractéristique marquante de cette conception réside dans la primauté de l'œuvre sur l'homme, du produit sur le producteur. En effet, l'éternité a un statut tellement central dans l'échelle des valeurs, que c'est elle qui confère la dignité : l'homme, en tant que mortel, a moins de valeur que ce qui lui subsiste, le producteur a moins de valeur que ce qui dure après lui : « je m'intéressais moins à eux qu'à la perfection du gâteau de miel auquel ils avaient tout le long du jour collaboré » (SaintExupéry, 1948, p. 43).

Enfin, la vocation de l'homme est de durer, et la création est le moyen d'atteindre cette vocation comme on l'a dit. Puisque leur vocation, plus grande que les êtres, les définit, l'homme se définit par son activité créatrice : «L'homme, disait mon père, c'est d'abord celui qui crée. » (Saint-Exupéry, 1948, p. 59). Pour Saint-Exupéry, l'homme est donc moins homo sapiens qu'homo faber. On peut soulever le paradoxe d'une telle assertion en disant que le travail comme création définit l'homme, mais le détruit en tant qu'il le définit. En effet, le travail fait de l'homme ce qu'il est, et défait l'homme par le même aspect, à savoir sa capacité à permettre à l'homme de durer. En cela, le travail tel que conçu par Saint-Exupéry assume le caractère ambigu que l'on reconnaît aujourd'hui à cette notion : moyen de s'épanouir en tant qu'homme, c'est davantage l'héritage de Marx (Méda, 2004, p. 18), et en même temps passage obligatoire, quoique contraignant et pénible, pour accéder à autre chose (la richesse pour Smith; Méda, 2004, p. 18). Arendt se fait l'écho de cette ambivalence qui existe au sein même de la pensée de Marx : «L'attitude de Marx à l'égard du travail, c'est-à-dire à l'égard de l'objet central de sa réflexion, a toujours été équivoque. Alors que le travail est une 
"nécessité éternelle imposée par la nature", La plus humaine la plus productive des activités, la révolution selon Marx n'a pas pour tâche d'émanciper les classes laborieuses, mais d'émanciper l'homme, de le délivrer du travail ; il faudra que le travail soit aboli pour que le domaine de la liberté supplante le domaine de la nécessité. Car "le domaine de la liberté ne commence que lorsque cesse le travail déterminé par le besoin et l'utilité extérieure", lorsque prend fin à la "loi des besoins physiques immédiats" » (Arendt, 2014, p. 151).

Mais, chez Saint-Exupéry, ce paradoxe n'est qu'apparent, puisque ce n'est pas réellement sous le même aspect que le travail détruit et construit l'homme : c'est bien en temps qu'il permet à l'homme de durer que le travail fait de l'homme ce qu'il est. Mais c'est en temps qu'échange, échange certes impliqué, et impliqué nécessairement par sa capacité à permettre de durer, qu'il défait l'homme : le travail, quoique sauvé de la contradiction, n'en conserve pas moins son statut ambigu.

Cependant, il faut noter que la valeur constructive du travail n'est pas systématique : «qui peut grandir dans l'esclavage, la cruauté, et l'abrutissement d'un lourd travail ? (Saint-Exupéry, 1948, p. 90). Il y aurait lieu ici de thématiser sur un registre qui s'inspirerait de la pensée d'Arendt, une distinction entre le travail créateur, celui par qui on se reconstruit dans l'œuvre, et le travail, qui détruit sans permettre de se reconstruire dans l'œuvre.

La troisième implication regarde l'empire comme bien commun. On a vu comment l'image de l'empire servait de véhicule pour faire atteindre l'éternité. En creusant plus loin, il apparaît comme ce qu'on pourrait appeler un bien commun, non pas comme un bien matériel, ou somme de biens matériels communs à tous les hommes de cet empire, mais comme la finalité commune à chacun de hommes et à l'ensemble des hommes, commune au tout et à la partie, et qui donne sens, oriente chaque action, chaque œuvre.

Il est ce vers quoi l'on tend, ce vers quoi l'on est porté par le désir, il est « l'invisible mamelle qui les allaitait nuit et jour, car l'empire t'alimente le cœur comme t'alimente de son amour et change pour toi le sens des choses la bien-aimée qui loin de toi s'est endormie et repose cependant comme morte. » (Saint-Exupéry, 1948, p. 71). Il change les choses comme le temple qui est sa finalité change l'œuvre du tailleur de pierre.

Ce désir qui porte l'homme vers sa finalité qui est son épanouissement, son accomplissement, peut être inconscient. Les hommes «méconnaissent» (Saint-Exupéry, 1948, p. 71) cette invisible mamelle. Ils ignorent que c'est l'empire qui donne sens, que c'est lui qu'ils désirent et qu'ils servent, cet invisible nœud qui noue tous les objets, et par eux tous ceux qui les créent, en les élevant à faire partie d'un tout plus grand qu'eux, comme une simple pierre quand elle fait partie d'un temple magnifique : «mais je n'écoutai point la réponse, sachant qu'il se tromperait et me parlerait de l'argent gagné ou du 
repas qui l'attendait ou du repos. Ne sachant point que son bonheur était de se transfigurer en babouches d'or. » (Saint-Exupéry, 1948, p. 48).

L'empire a donc deux caractéristiques : il est une finalité, au sens de l'objet d'un désir (conscient ou inconscient), au sens de ce qui permet d'accomplir. Cette finalité, en tant que polarisatrice de toutes les œuvres, a aussi le pouvoir d'unir tous les hommes comme la construction d'un citadelle unit ses bâtisseurs : « Forces-les de bâtir une tour, et tu les changeras en frères » (Saint-Exupéry, 1948, p. 58).

\section{DU POEME AU DISCOURS EXPLICITE}

On peut avoir un doute sur la part de l'esthétique dans cette expression poétique de la pensée livrée dans Citadelle. Rappelons que nous nous situons dans une démarche méthodologique inspirée du schème herméneutique de Sabot : comme nous l'avons dit plus haut, dans ce schème, l'œuvre littéraire contient une vérité. Le penseur est amené à « rappeler cette vérité à l'ordre du concept, selon un process interprétatif qui opère en quelque sorte la traduction du langage littéraire, où cette vérité est inapparente, comme dissimulée à elle-même par son mode propre de manifestation, dans le langage philosophique, où elle reçoit son élucidation spéculative» (2002, p. 54, souligné dans le texte). Or, cela suppose que Saint Exupéry ait voulu dire quelque chose du travail, en l'occurrence, certes sur un mode poétique, mais pour transmettre sa pensée réelle. Par conséquent, notre travail herméneutique doit s'appuyer sur la certitude que les textes de Citadelle sur le travail ne font pas la part belle à l'esthétique, sans vouloir systématiquement dire quelque chose.

Certes, l'auteur a écrit « je crois si fort à la vérité de la poésie » (1994, p. 579). Mais pour s'en assurer, on peut comparer la pensée du « seigneur berbère » exprimée dans Citadelle sur le travail à un autre texte de Saint-Exupéry : un discours prononcé à de jeunes américains (2015), en décembre 1941, alors qu'il travaille à la rédaction de Citadelle. On y retrouve, sous un mode non-poétique, la pensée de Citadelle. Sur le travail à «double-fond», et l'attention détournée de l'échange plus profond que celui qui se joue entre l'effort et le salaire, Saint-Exupéry donne son explication: «la nécessité impérieuse ou s'est trouvée l'humanité d'arracher l'homme à l'esclavage, en lui assurant le fruit de son travail, a fait porter l'attention sur le travail valeur d'échange. Sur le travail marchandise. Mais nous ne devons pas oublier qu'un des aspects essentiels du travail n'est pas le salaire qu'il procure à l'homme, mais l'enrichissement spirituel qu'il lui apporte » (p. 69). Les catégories de Marx sont utilisées pour décrire l'échange superficiel, qui retrouve ici son aspect idolique, au détriment de sa valeur iconique. En effet, cet échange visible détourne l'attention que l'on pourrait porter sur l'échange invisible qui s'y opère. L'expérience personnelle du pilote de ligne sert d'illustration : «j'ai touché un salaire. Je pouvais chaque mois me procurer quelques-uns des biens souhaités, avec l'argent de mon salaire» $(2015$, p. 69). Il s'agit de l'échange superficiel, avec le cycle improductif travail consommation. Mais le travail donne plus que les moyens de consommer : « si mon travail de pilote 
de ligne ne m'avaient rien assuré d'autre que ces avantages quelconques, pourquoi l'aurais-je tant aimé ? Il ma donné bien plus.» (2015, p. 69). Pour faire un pas de plus dans le raisonnement avec Saint-Exupéry, ajoutons que si ce qui est reçu est plus que du salaire, ce qui est donné est plus qu'un simple effort : « mais il me faut reconnaître qu'il m'a enrichi véritablement, là seulement où j'ai donné plus que je n'ai reçu» (p. 69). Ce supplément donné n'est pas un supplément d'effort. Ce n'est pas plus grand en degré, mais en nature. Pour exprimer ce qui est donné alors de plus que ce qui mérite salaire, Saint-Exupéry emploi le terme de «sacrifice», « et par sacrifice je n'entends pas le renoncement aux bien de la vie, ni le désespoir dans la pénitence. Par sacrifice, j’entends le don gratuit. Le don qui n'exige rien en échange» (p. 69). L'étape suivante du raisonnement est la conséquence de ce don : « ce n'est pas ce que vous recevez qui vous fonde. C'est ce que vous donnez à la communauté qui fonde la communauté et l'existence d'une communauté enrichit votre propre substance» (p. 69). Voilà l'échange plus profond, sous l'échange d'un salaire (donc de la consommation possible) contre un effort avec un employeur, un client, on a l'échange d'un don gratuit de soi (sacrifice) «contre» (il ne s'agit pas d'un échange conçu sur le modèle des échanges marchands, bien entendu) une croissance humaine avec la communauté (p. 69). De ce point de vue, « il convient d'enrichir la communauté parce qu'elle seule à son tour enrichit l'homme [...]. Une cathédrale est faite de pierres. Les pierres composent la cathédrale. Mais la cathédrale ennoblit chacune des pierres » (p. 68). C'est donc bien par l'intermédiaire de la communauté (l'empire de Citadelle) que le travail est un don au travailleur d'une humanité augmentée.

Il est particulièrement intéressant de noter le parallèle avec un autre texte, fondateur en théorie des organisations, qui mobilise l'image de la cathédrale pour cristalliser le lien entre travail individuel et communauté. Dans The practice of management, Peter Drucker, instaure le management by objectives, pour répondre aux exigences de la décentralisation des grands groupes américains. Dans ce livre, il fait référence également à la construction d'une cathédrale, pour prouver une idée parente de celle de Saint-Exupéry. Il décrit une rencontre avec trois tailleurs de pierre (1993, p. 122): «le premier répond : "je gagne ma vie", le second, tout en martelant, dit: "je suis en train de faire la meilleure taille de pierre de la région" ; le troisième leva au ciel ses yeux dans un regard de visionnaire et dit "je bâtis une cathédrale" ${ }^{2} »$. Drucker montre que le plus problématique est le second, car, grisé par son «workmanship », la qualité de son travail de spécialiste, il risque d'en oublier que son travail est au service de la performance globale: «La qualité de travail spécialisé doit être encouragée dans une entreprise, mais elle doit toujours être reliée aux besoins du tout ${ }^{3} »(1993$, p. 122). Les deux paragraphes du texte de Drucker montrent ce qu'il entend par là : «Ce dont a besoin l'intérêt d'une

\footnotetext{
${ }^{2}$ The first replied, "I am making a living." The second kept on hammering while he said, "I am doing the best job of stonecutting in the entire country." The third one looked up with a visionary gleam in his eyes and said, "I am building a cathedral".

3 "Workmanship must be encouraged in the business enterprise. But it must always be related to the needs of the whole".
} 
entreprise est un principe de management qui donne toute sa valeur à la force individuelle et à la responsabilité, et en même temps donne une direction commune de vision et d'effort, met en place le travail d'équipe et harmonise les buts de l'individu avec le bien du tout. Le seul principe qui puisse faire cela est le management par objectifs et le self-control. Il fait du but commun l'objectif de chaque manager $^{4} »(1993$, p. 136). Ce parallèle est intéressant, en ce qu'il permet de montrer la radicalité de la pensée de Saint-Exupéry, sur un sujet similaire, puisque chez les deux auteurs, il s'agit d'unifier la diversité des activités. Le texte de Saint-Exupéry, d'une certaine façon, va plus loin, puisque dans l'exemple de Drucker, ce n'est que le fruit du travail (la pierre taillée) qui doit trouver harmonieusement sa place grâce au but commun. Dans le texte de Saint-Exupéry, au contraire, c'est le travailleur lui-même qui est la pierre, c'est à dire qui, par et dans son œuvre, se trouve inséré dans une communauté. S'il fallait chercher la source de cette image radicale chez Saint-Exupéry, il faudrait sans doute se tourner vers ce passage de l'épître de Saint Pierre: «Vous mêmes comme des pierres vivantes, entrez dans la structure de l'édifice, pour former un temple spirituel » (I Pierre, 2, 5).

Ce court texte didactique de Saint-Exupéry constitue donc une synthèse de sa conception du travail. Cependant, l'un des éléments de Citadelle ne s'y retrouve pas. Il s'agit de la question de la temporalité. L'échange plus profond se fait avec la communauté (qui correspond à l'empire dans Citadelle). Dans Citadelle, l'échange se fait avec le temps, duquel on soutire l'éternité. Mais il n'y a pas incompatibilité entre les deux expressions, dans la mesure ou le temps et l'espace sont intriqués dans le désert du seigneur berbère. La notion de cérémonial permet de le manifester. Le cérémonial peut se définir comme un agencement chronologique qui structure une temporalité. Dans Citadelle, il est l'architecture même des choses, leur source d'unité : «Je ne connais rien au monde qui ne soit d'abord cérémonial car tu n'as rien à attendre d'une cathédrale sans architecture.» (1948, p. 353). Et plus loin : «l'objet lui-même est cérémonial de ses parties. » (p. 353). C'est aussi un cérémonial qui va unir les hommes dans la communauté : «les individus [...] ne sont plus que pierres en vrac si tu ne fondes pas dans ton empire un cérémonial des hommes » (p. 353). L'empire, dans sa structuration, déborde l'individu de toute part, en ce qu'il lui préexiste, et perdure après lui, notamment grâce à lui. C'est en cela que l'empire (la communauté) est la promesse de l'éternité de l'homme. Donc dans Citadelle, la dimension temporelle couvre la dimension de la communauté, dans un lien mystérieux.

On peut en conclure que dans ce texte, en quelque sorte, Saint-Exupéry nous dispense de la traduction qu'exige le schème herméneutique. Il nous la fournit, du moins dans ses grandes lignes. Cette traduction conforte donc la lecture que nous avons faite de Citadelle.

\section{LES APPORTS POUR LA GESTION}

\footnotetext{
${ }^{4}$ «What the business enterprise needs is a principle of management that will give full scope to individual strength and responsibility, and at the same time give common direction of vision and effort, establish team work and harmonize goals of the individual with the common weal. The only principle that can do this is management by objectives and self-control. It makes the common weal the aim of every manager".
} 


\subsection{Articuler les trois dimensions du travail}

L'apport principal de la pensée de Saint-Exupéry pour la gestion réside, selon nous, dans l'articulation entre les trois dimensions du travail, telles que développées par Gomez (2013). En effet, celui-ci développe une thèse selon laquelle le travail « constitue une triple expérience : une expérience subjective par ce que, quel qu'il soit, le travail est toujours effectuée par un sujet singulier : quelqu'un travaille ; une expérience objective parce qu'il débouche sur la production d'un objet matériel, un bien ou un service : le travail fabrique quelque chose; une expérience collective, parce qu'on ne travaille jamais seul mais soit avec d'autres, soit pour d'autres: le travail s'inscrit dans une communauté sociale » (2013, p. 173). Autrement dit, le travail fait quelque chose (objectif), il me fait quelque chose (subjectif), et il fait communauté (collectif). La financiarisation des entreprises, selon Gomez, cause une «hypertrophie du travail objectif» (p. 188) qui occulte les autres dimensions, d'où l'invisibilité du travail. Il propose donc de donner davantage de place à la dimension subjective par la reconnaissance de la gratuité (p. 208), et à la dimension collective par la fierté du chef envers ses collaborateurs (p. 218), en plus de donner du sens au travail pour valoriser à sa juste mesure la dimension objective (p. 214)

Ce que vient apporter la pensée de Saint-Exupéry à cette réflexion relève non pas tant de l'une ou l'autre dimension du travail, mais de leur articulation. En effet, ce que permet de montrer l'auteur de Citadelle, c'est l'inter-inclusion des trois dimensions du travail entre elles. Par conséquent, il permet de conforter la thèse de Gomez, puisque dès lors, arrêter son regard à l'aspect objectif dénature plus profondément le travail. Pour montrer les conséquences que cela peut avoir, on pourrait dire que dans la synthèse de sa proposition managériale, Gomez s'exprime en superposant les trois recommandations : « D'abord [...] laisser une juste place à la gratuité [...]. Ensuite, [...] donner du sens au travail. Enfin, [...] être fier...» (pp. 220-221). Nous affirmons que le recours à la pensée de Saint Exupéry permettrait de transformer cette accumulation en concaténation. Ce qui donnerait quelque chose comme : laisser une place à la gratuité pour donner du sens au travail, et par conséquent être fier... Cette concaténation évincerait d'emblée toute velléité de rejeter de façon idéaliste la dimension objective du travail sous-prétexte qu'elle a été hypertrophiée par la financiarisation. Elle a aussi l'avantage d'expliquer le malaise de certaines conceptions du bonheur au travail qui valorisent la dimension subjective sans lien avec la dimension objective, amenant à déresponsabiliser les travailleurs, c'est à dire à les désolidariser de la contribution sociétale de leur travail : les salariés heureux d'une manufacture de bombes atomiques.

Comment cette articulation peut-elle être pensée grâce à la pensée de Saint-Exupéry ? Pour le dire en une phrase, il faudrait dire que ce qui est fait par le travail, c'est-à-dire l'objet (objectif), est un vecteur qui va permettre au travailleur lui-même, de s'insérer dans la communauté (collectif), qui par cela même va lui rendre plus que ce qu'il a apporté dans l'œuvre, et ainsi l'augmenter en tant que sujet 
(subjectif) du travail. L'articulation entre les trois dimensions du travail se fait donc ainsi: la dimension objective est l'occasion du déploiement de la dimension collective qui ipso facto met en lumière la dimension subjective.

Il est évident que cet enchaînement ne fait l'objet d'étapes chronologiquement distinctes. Il s'agit en fait de trois facettes d'un même objet. C'est en faisant l'œuvre, et par le fait même de faire l'œuvre que je m'insère dans l'empire, et l'insertion dans l'empire est elle-même cette augmentation du sujet travaillant en ce qu'il devient «l'homme d'une patrie, d'un métier, d'une civilisation, d'une religion » (2015).

Une fois précisé que les étapes de la concaténation ne sont pas des étapes chronologiques mais logiques, il est nécessaire de les décrire. Le premier moment consiste en la faction d'une œuvre. Le travailleur fournit un effort pour aboutir à un résultat, qui lui mérite salaire, donc possibilité de consommation. Il s'agit en fait de l'échange superficiel, visible du travail. A ce titre, arrêter son regard à cette seule dimension objective, comme le font les entreprises financiarisées revient à poser cet échange dans un rapport idolique avec le travailleur. Cette dimension, plutôt que d'ouvrir sur une dimension plus profonde, arrête à elle-même le regard, voilant ce qu'elle doit dévoiler. Le regard est alors renvoyé au seul horizon émergé que semble offrir le travail : la consommation.

Mais le travail est à double fond : au cœur même de cet factivité d'une œuvre échangée contre un salaire pour consommer se trouve un autre échange. La fabrication des babouches d'or est l'occasion d'une «fabrication» de l'empire (collectif), qui de ce fait «fabrique» le savetier (subjectif). L'échange plus profond est donc le deuxième et le troisième moment du travail. Dans le deuxième moment, la dimension collective du travail, l'œuvre contribue à former l'empire, par ce que le travailleur y met de "sacrifice ", de don de soi. Dans le troisième moment, l'empire augmente le travailleur de tout ce que les autres travailleurs ont donné d'eux-mêmes, et cela par le don même que fait le travailleur, et non dans un second temps.

Il est sans doute possible de comprendre cette tripartition chez Saint Exupéry, en la voyant dans un état diffracté, telle qu'elle apparaît dans la structure de la vita activa qu'élabore Hannah Arendt. Chez elle, au sein de la vita activa, le travail s'oppose à l'œuvre en ce qu'il s'inscrit dans un cycle improductif de métabolisme, non spécifiquement humain (on parle bien d'animal laborans), comparable à la face émergée de l'échange travail/consommation que l'on peut interpréter dans les écrits de Saint-Exupéry. Le régime de l'œuvre, au contraire, va briser cette circularité pour installer le monde d'objets dans la durabilité. Elle ne parle pas d'éternité comme le fait Saint-Exupéry dans son langage poétique, mais cette durabilité n'est pas sans apporter en retour à l'homme qui les construit: «A ce point de vue, les objets ont pour fonction de stabiliser la vie humaine, et [...] leur objectivité tient au fait que les hommes en dépit de leur nature changeante, peuvent recouvrer leur identité dans 
leurs rapports avec la même chaise, la même table. » (2014, p. 188). Le rôle cardinal que joue la durabilité de l'œuvre se manifeste dans le fait qu'elle sert de différence spécifique avec le travail :

« Il semble que la distinction du travail et de l'œuvre [...] ne soit plus en effet une différence de degrés, si l'on ne prend pas en considération le caractère d'objet-de-cemonde de la chose produite : son emplacement, sa fonction, la durée de son séjour dans le monde. La distinction entre un pain, dont la "vie moyenne" ne dépasse guère une journée, et une table, qui survit aisément à plusieurs générations humaines, est certainement beaucoup plus nette est plus décisive que la différence entre un boulanger et un menuisier » (2014, p. 139).

On retrouve alors le rôle clé que joue l'éternité, de façon étonnante au premier abord, dans la question du travail chez Saint-Exupéry. En revanche, pour Arendt, contrairement à Saint-Exupéry, la dimension collective doit être trouvée par l'homme dans l'action, «la seule activité qui mette en rapport les hommes, sans l'intermédiaire des objets ni de la matière... » (2014, p. 41). C'est donc en contournant les nécessités du travail (qui met l'homme en rapport avec la nature), et au delà de la logique de l'œuvre (qui met l'homme en rapport avec le monde d'artefacts) que l'homme peut entrer en rapport avec les autres hommes. On voit que Saint-Exupéry, pour parler en terme arendtiens, fait porter au travail les fonctions du travail, de l'œuvre et de l'action réunis. Il faudrait aller plus loin pour établir les points de divergences entre les deux œuvres, mais à ce stade, la comparaison avec la tripartition de la vita activa thématisée par Arendt, malgré ses limites, permet de voir la distinction des trois moments de la démarche laborieuse chez Saint-Exupéry.

A partir de là, on peut dégager quelques pistes de réflexion qui éclairent trois éléments du contexte économique et managérial actuel : le sens du travail, le questionnement de l'implication des cycles d'emploi courts, et la prise en compte des parties prenantes.

\subsection{Pour éclairer la dimension subjective : le sens du travail}

Le sens du travail fait l'objet de recherches en gestion, et pour cause, il est parfois difficile, dans un contexte économique fragmenté, aux temporalités multiples, de trouver ce sens. Pourtant l'enjeu est capital, car le travail semble jouer un rôle important dans l'attribution d'un sens global à l'existence : « Sans travail au sens large du terme, l'individu connaîtrait une situation de détresse que Frankl (1969) qualifie de spirituelle» (Berthoin Antal et Frémeaux, 2013, p. 5). Se définissant comme une activité qui a un but, le travail répond à ce besoin humain de chercher un sens : " Il se peut donc que tout individu ait besoin d'entretenir un rapport spirituel à son travail (Dyck and Schroeder, 2005), même s'il n'en a pas conscience ou n'utiliserait pas le terme de spiritualité pour évoquer son besoin de donner du sens au travail. » (Berthoin Antal et Frémeaux, 2013, p. 5). 
Anthony voit déjà chez Marx cette analyse : «Bien que Marx les ait énoncées sous une forme beaucoup plus complexe, les caractéristiques essentielles de cette conception sont relativement simples : a) le travail devrait avoir un sens, ou il en avait u n autrefois ; b) ce sens fondamental a été détruit. » (1980, p. 446). Aujourd'hui, on affirme que beaucoup éprouvent des difficultés à percevoir le sens leur travail (Méda, 2010, p. 135).

Il semble que la conception du travail qui affleure dans Citadelle de Saint-Exupéry contribue à penser un travail sensé. En effet, le point de vue à partir duquel Saint-Exupéry regarde l'échange modifie la perspective par rapport au travail.

Un bref constat préalable permet de mieux montrer l'apport de Saint-Exupéry à la question. Quand on dit que certains travailleurs ont des difficultés à percevoir le sens de leur travail, on sous-entend par là que le lien entre l'activité de travail et la réussite de la vie personnelle n'est plus évident. Ce qui manque, c'est une unité entre la finalité de l'activité de travail et la finalité de vie personnelle. L'aspect directionnel du contenu sémantique du mot sens invite à cette lecture d'unification des directions de vie.

Or, la vision de Saint-Exupéry fait précisément de la vie l'objet du travail : l'unité entre la vie et le travail n'est pas conjoncturelle, elle est structurelle. En reprenant les éléments du tableau ci-dessus, on voit que ce qui est émis, dans une conception économique du travail comme échange, ce qui est mis dans la balance, est simplement un « effort» quoiqu'entendu au sens large, tandis que pour SaintExupéry, c'est la vie qui est jouée. C'est elle qui est l'objet du travail. L'objet de l'activité du travailleur, en quelque sorte, c'est lui-même. Donc il n'y a plus de dichotomie entre ce que l'on fait et ce que l'on est : « je veux fonder la noblesse de mes guerriers et la beauté des temples contre quoi les hommes s'échangent et qui donnent sens à leur vie » (Saint-Exupéry, 1948, p. 79).

La pensée de Saint-Exupéry semble radicale, en ce qu'elle identifie réussite de la vie et travail accompli. Il faut prendre cette pensée radicale comme une idée régulatrice qui permet de sortir de la séparation radicale entre l'homme et ce qu'il produit, séparation thématisée par Marx sous le terme d'« aliénation ».

Saint-Exupéry répond à cette séparation du travail par la confusion des domaines de l'agir et du faire, de l'œuvre réussie et de l'action bonne.

La distinction entre action transitive et action immanente permet de penser cette opposition. Une action transitive a son terme en dehors du sujet qui la pose. Une action immanente a son terme dans le sujet qui la pose. Le travail est habituellement conçu comme une action transitive, dans laquelle le travailleur transforme en dehors de lui quelque chose qui n'est pas lui. Au contraire, à l'image de l'arbre qui produit son fruit, fruit qui est encore l'arbre, Saint-Exupéry conçoit le travail comme une 
activité immanente, produisant un autre moi : les babouches (en tant que participation à l'empire) sont le savetier, qui de ce fait, en les créant, ne sort pas de lui-même, donc pose une action immanente. Cette immanence se caractérise par la fécondité, avec sa temporalité propre («Je veux qu'ils se nourrissent, pareils à des fruits qui s'achèvent, de silence et de lenteur. Je veux qu'ils soient semblables à la branche d'olivier, celle qui attend» Saint-Exupéry, 1948, p. 23), par opposition à l'efficacité qui accompagne la transitivité de l'activité de travail.

Une piste pour retrouver le sens du travail serait donc de retrouver l'aspect immanent qui existe dans le travail. Il ne s'agirait pas d'éliminer l'aspect transitif, qui existe toujours, mais de regarder le travail avec ce regard d'immanence, qui fait que l'on met de soi dans ce que l'on fait, même si certaines structures ne disposent pas leurs salariés à voir son travail sous cet angle-là. L'implication souhaitée par les entreprises pour leurs salariés ne peut se faire sur le mode de la transitivité, aliénante et destructrice, mais davantage sur le mode de l'immanence.

On peut ajouter que le travail peut retrouver un sens avec Saint-Exupéry en tant qu'il s'oppose à la possession. Dans Citadelle, on trouve de nombreux passages qui renvoient tous, d'une façon ou d'une autre, à l'idée suivante : la possession pour elle-même détruit l'homme.

Ce n'est pourtant pas au nom d'une justice sociale que la possession est rejetée, comme c'est le cas par exemple dans la pensée de Proudhon (1873, p. 17). La possession est rejetée parce qu'elle enferme dans l'égoïsme : «Ceux-là, vous les avez vaincus, car ils s'enfermaient dans leur égoïsme, satisfaits par leurs provisions. [...] Ils croupissaient dans l'illusion du bonheur qu'ils tiraient de biens possédés. Alors que le bonheur n'est que chaleur des actes et contentement de la création » (Saint-Exupéry, 1948, p. 51-52). La conséquence de la possession et de la consommation de ce qui n'est pas le fruit de son travail a des conséquences néfastes :

Ceux qui n'échangent plus rien d'eux-mêmes et reçoivent d'autrui leur nourriture, fût-elle la mieux choisie et la plus délicate, ceux-là mêmes qui, subtils, écoutent les poèmes étrangers sans écrire leurs propres poèmes, jouissent de l'oasis sans la vivifier, usent des cantiques qu'on leur fournit, ceux-là s'attachent d'eux-mêmes à leurs râteliers dans l'étable et, réduits au rôle de bétail, sont prêts pour l'esclavage.

Ainsi, «les provisions sont nécessaires, mais plus dangereuses que la famine » (Saint-Exupéry, 1948, p. 52). Elles sont dangereuses quand elles ne sont pas gagnées par le travail : «Forces-les de bâtir une tour, et tu les changeras en frères, mais si tu veux qu'ils se haïssent, jette-leur du grain. » (Saint-Exupéry, 1948, p. 58)

On peut donc dire que le travail ne s'oppose pas tant à la possession qu'il n'est régulé par elle et qu'il ne la régule. Cette régulation mutuelle du travail et de la possession fait que le travail qui est 
exclusivement pour la possession n'est pas achat d'éternité. Cette régulation mutuelle serait un cercle stérile si elle ne s'adossait pas à une conception du travail comme ayant une finalité double. D'une part, l'utilité immédiate (possession, consommation), de l'autre une finalité médiate (l'empire). Ces deux finalités ne sont pas concurrentielles pour Saint-Exupéry, même si parfois celui qui œuvre le croit, comme le savetier qui croit gagner sa vie alors qu'il se transfigure en babouche d'or, donc en part d'empire.

Ces réflexions diffuses dans le texte de Saint-Exupéry, sont autant d'appels à trouver une mesure au travail et à la consommation. On peut peiner à vouloir mesurer le travail (Concialdi, 2009, p. 177). Par ailleurs, «Les dépenses de consommation des ménages augmentent de 3,2 \% par an en moyenne entre 1960 et 2008 (soit une très forte augmentation du volume de consommation) » (Chessel, 212, p. 23). Ces deux éléments, travail et consommation semblent attendre une régulation externe (par des normes pour le travail), par la nécessité pour la consommation. Mais l'on n'attend pas d'eux une régulation interne. La pensée de Saint-Exupéry invite à penser une auto-régulation mutuelle du travail et de la consommation. Si l'on retrouvait le lien régulateur entre l'un et l'autre, on atteindrait une condition pour rendre sens au travail.

\subsection{L' «éternité » pour questionner les cycles d'emploi courts}

La mobilité a un statut ambigu dans le contexte actuel. Notais et Perret (2012) le notent bien, en parlant de «l'univers organisationnel où la mobilité est généralement parée de nombreuses vertus, et en particulier posée comme une pierre angulaire de la gestion efficace des ressources humaines et un outil au service de la dynamique organisationnelle. Pourtant, pour l'individu, la mobilité n'est pas un phénomène anodin et banal» (2012, p. 122). John Urry voit dans le monde mobile contemporain autant « de nouveaux dangers, effrayants, que [d'] opportunités nouvelles pour ceux qui ont choisi le risque et la mobilité » (Urry, 2005, p. 27). Au delà de la mobilité professionnelle, Barrère et Martucelli (2005) dans un contexte de recherche plus large, brossent un portrait de l'imaginaire contemporain de la mobilité, et montrant les inflexions subies par la constellation moderne de représentations autour de la mobilité. Ils concluent en écrivant, au sujet de la mobilité dans l'imaginaire contemporain: «Dégagée de tout devenir héroïque et collectif, elle se souvient pourtant de ses anciennes promesses et les continue d'une certaine manière. L'imaginaire de la mobilité est alors sous le double signe de la déception, voire de la démystification, et de la réinvention, de la transformation créatrice » $(2005, \mathrm{p}$. 62). Pour ce qui relève plus spécifiquement du management, Aubert et Gaulejac (2007) voient dans l'exigence d'adaptabilité permanente une composante de «la thématique commune du discours managérial »:

Il faut être mobile, disponible au changement, capable de s'adapter à des situations différentes, prêt à affronter l'incertitude, mais une incertitude qui ne laisse pas prise au 
doute sur le bien-fondé de l'action. Les fluctuations du marché, l'accélération des changements technologiques l'introduction de la flexibilité comme norme d'organisation du travail... autant d'éléments qui contribuent à insister sur la mobilité des hommes et leur capacité d'adaptation. Le changement devient une valeur en soi. La résistance au changement est a priori négative (2007, p. 104-105).

On voit donc que la mobilité n'est pas nécessairement une valeur perçue comme positive. Des cycles d'emploi de trois ans, cinq ans, ponctuent parfois les carrières professionnelles, qui peuvent apparaître a posteriori comme décousues. La conjoncture évolue très vite, poussant les dirigeants à restreindre leur vision à un terme toujours plus court, générant des changements organisationnels à un rythme que les salariés ont parfois du mal à suivre (Borchgrave, 2006, p. 31). Le risque d'une telle mobilité est l'affaissement de la perception des responsabilités (à force de prendre des décisions dont on aura parfois pas à répondre, puisque les conséquences de ces décisions apparaîtront une fois seulement que le décideur aura changé de poste), et l'effacement de la conscience de la personne de pouvoir changer les choses à son niveau.

Le rapport du temps au travail selon Saint-Exupéry permet d'éclairer cette problématique, dans la mesure où la responsabilité de celui qui œuvre est fonction de son implication vitale dans l'œuvre accomplie, implication ineffaçable : «cet effort t'apparaît stérile à toi, aveugle, qui te tiens le nez contre, mais recule-toi. Considère de plus loin le mouvement de ce quartier de ville...» (SaintExupéry, 1948, p. 59). Chacun est responsable de l'empire, et le travail accompli demeure au niveau de l'empire, même une fois les babouches usées. On peut donc retrouver le sens du travail à long terme, donc chaque tâche, chaque mission, chaque cycle d'emploi, s'unifie avec les autres dans une carrière de laquelle le regard perçoit d'abord l'unité : chaque tâche, chaque mission, chaque cycle d'emploi a la même fin. L'échange pour durer permet donc de questionner les cycles de mobilité très courts et de leur donner une unité responsabilisante.

6.4 Un bien commun pour les parties prenantes : la dimension collective du travail à la lumière de l'empire.

Dans la réflexion sur la théorie des parties prenantes, il est difficile de penser une prise en compte vraiment objective des demandes des parties prenantes : «On pourrait aussi qualifier la RSE de sorte de monologue adressé aux agents sociaux comme s'il s'agissait d'un dialogue alors qu'ils n'ont rien demandé » (Pesqueux, 2011, p. 51). Pesqueux explique qu' «il y a en fait plus co-construction des informations de restitution aux parties prenantes qu'un véritable dialogue » (2011, p. 48).

La conception du travail de Saint-Exupéry apporte des pistes de réflexion pour poser ce problème autrement. L'empire, ce bien commun, diffère d'un intérêt général, en suivant Saint-Exupéry. En effet, 
dans la conception du travail de Saint-Exupéry, on s'échange contre quelque chose de plus grand mais, au final, on s'y retrouve : c'est l'idée du bien commun, par opposition à l'intérêt général dans lequel on ne se retrouve pas. L'empire est le lieu d'épanouissement de la personne, le lieu où il est grand: «Tu n'es grand qu'à cause de l'empire que tu sers » (Saint-Exupéry, 1948, p. 56). Cette réalité se manifeste dans un passage d'une radicalité dérangeante. En parlant à un homme à qui un autre sauverait la vie, il écrit :

Et toi, si tu exprimes trop fort ta reconnaissance, c'est que tu manques à la fois et de modestie et d'orgueil. Car l'important qu'il a sauvé, ce n'est point ton petit hasard personnel, mais l'œuvre à laquelle tu collabores et qui s'appuie aussi sur toi. Et comme il est soumis à la même œuvre, tu n'as point à le remercier. Il est remercié par son propre travail de t'avoir sauvé. C'est là sa collaboration à l'œuvre » (Saint-Exupéry, 1948, p. 55).

En définitive, on pourrait dire que servir l'autre, c'est servir l'œuvre commune, donc c'est se servir. La médiation de l'empire fait du service à l'autre un moyen de se réaliser.

Dans cette optique, c'est-à-dire en regardant les choses à partir du bien commun à laquelle renvoie l'image de l'empire comme finalité commune, la prise en compte des objectifs des parties prenantes, en tant que ces parties prenantes visent également le bien commun, est un moyen pour l'organisation d'atteindre sa finalité, comme le pose la conception intégrative de la RSE (Perez, 2005, p. 36).

La lecture de Citadelle de Saint-Exupéry donne donc trois pistes de réflexion pour la gestion: contribuer à rendre au travail son lien vital avec la réussite de la vie, questionner les cycles courts d'emploi et les replacer dans une perspective à long terme favorable à la conscience de sa responsabilité, et enfin proposer une conception du bien commun favorable à une théorie des parties prenantes vraiment « dialogale».

\section{CONCLUSION : LES LIMITES DE L'APPORT DE SAINT-EXUPERY POUR LA GESTION}

La pensée de Saint Exupéry permet donc de dégager une théorie de l'articulation des trois dimensions du travail en ce que la dimension objective est le niveau de surface de l'échange constitué par le travail, alors que le collectif et le subjectif sont, au cœur de la dimension objective, le lieu de l'échange souterrain entre le don de soi du travailleur qui l'inscrit dans l'empire et le don par l'empire d'un statut supérieur à la simple individualité. De là, sont possibles trois apports pour la gestion mais on voit que dans les trois cas, ce que l'on proposer à partir d'une lecture de Saint-Exupéry, n'est pas de changer les choses, mais de changer de regard sur les choses. Les regarder à partir de la finalité de 
la vie (durer), de la finalité du travail (construire l'empire), et de la finalité commune à tous (bien commun). Cette finalité est ce qui permet de donner un sens, c'est-à-dire une direction au travail.

Au terme de ce travail, il est nécessaire de montrer les limites de la transposition opérée entre une pensée poétique et les sciences de gestion. Au-delà des limites propres à la traduction d'un texte littéraire en données conceptuelles propres à dialoguer avec les sciences de gestion, que nous avons soulignées au début de ce travail, on peut souligner deux points de vigilance dans l'application aux sciences de gestion, et trois limites propres à la pensée de Saint Exupéry lui-même.

Le premier regarde le lien entre l'activité professionnelle et la réussite de la vie. Il ne touche pas spécialement la pensée de Saint-Exupéry, mais toute pensée qui voudrait unifier le travail et la quête de bonheur. En effet, à proposer une alternative à la façon de fonctionner que nous avons décrites (perte de sens au travail, cycles courts de l'emploi, nécessité de prendre en compte réellement les parties prenantes, on s'expose à ce que Boltanski et Chiapello appellent le «désarmement de la critique » (2011, p. 257). Les auteurs décrivent comment le capitalisme «s'incorpore [...] une partie des valeurs au nom desquelles il était critiqué ». Ainsi de la thématique du bonheur au travail, qui, au départ pouvait être promu comme une régulation du productivisme outrancier, et peut servir aujourd'hui d'instrument pour intensifier l'exigence de productivité. Notre proposition d'une lecture par la pensée de Saint-Exupéry du travail pour unifier la réussite de la vie et l'activité professionnelle est, à cet égard ambigu : l'articulation entre les deux peut être vue comme une subordination de l'activité professionnelle à la réussite de la vie, mais on peut aussi la prendre comme une subordination de la réussite de la vie à l'activité professionnelle.

Le second point de vigilance concerne la prise en compte des parties prenantes. Selon Pesqueux, «l'éthique des affaires puis la RSE émergent avec la " crise des lois » dans le contexte de la mondialisation et de la déréglementation qui l'accompagne. Il ne s'agit donc pas d'une réponse à une demande sociale. C'est une "pseudonormalisation" (qui peut, à la limite, être vue comme propagande, alibi, justification idéologique, transgression discursive) de l'ordre du micro-politique (l'entreprise) et non dépourvue d'arrière-pensée publicitaire » (2011, p. 38). Dès lors, la pensée de Saint-Exupéry que nous proposons comme éclairage pour stimuler une réflexion alternative, pourrait être récupéré pour légitimer le système en place.

Enfin, trois limites quant à la pensée elle-même de Saint-Exupéry peuvent-être soulignées : La première regarde la fusion entre ce qu'on peut appeler la praxis et la poiesis. En effet, la tradition philosophique, depuis Aristote jusqu'à Hannah Arendt, a distingué nettement l'agir du faire. Le premier, dans l'éthique à Nicomaque, affirme que «production et action sont distinctes » (1990, p. 283). Dans l'une, en effet, le terme du devenir en question est extérieur au sujet : le lit fabriqué. En cela, la production et une activité transitive, car une forme transite hors du sujet pour s'imprimer dans 
une matière extérieure. Dans l'autre, le terme est dans l'agent lui-même : l'agent devenu plus ou moins vertueux après son action. On parle alors d'une activité immanente. L'un vise le bonum operis (bien de l'œuvre), l'autre vise le bonum operantis, le bien de l'agent. Cette distinction a l'avantage de délimiter le champ éthique du champ factif. Elle permet à Hannah Arendt de proposer une « révélation de l'agent» (2014, p. 231) dans autre chose que la réalisation d'une œuvre. Or, chez Saint Exupéry, le travail est, en tant que tel, la façon de devenir meilleur. C'est par le travail et dans le travail que le sujet devient plus grand. Les deux plans sont confondus pour ne former plus qu'un. D'une certaine façon, on y retrouve les mêmes caractéristiques, puisque les deux démarches font quelque chose dans l'agent (c'est le propre d'un habitus que d'être engendré par les actes correspondants, cf. Aristote, 1990, p. 88. C'est en forgeant que l'on devient forgeron). La production développe le tour de main, l'action bonne développe l'humanité. Mais chez Saint-Exupéry, ce n'est plus deux ordres différents : le travail devient l'horizon éthique de la vie humaine. Comment, dès lors, trouver un sens à sa vie en dehors du travail? En cela, il y a une certaine similitude avec la pensée de Simone Weil, qui donne également une valeur éthique, voire mystique, au travail.

Une autre limite peut-être soulignée, dans la conception du travail de Saint-Exupéry. Pour cela, il faut voir qu'il y a deux aspects de la dimension subjective du travail. Ces deux aspects qu'évoque Gomez sans les distinguer explicitement, méritent d'être mis en lumière. D'une part, Gomez écrit que la dimension subjective le travail «fabrique le sujet qui travaille» $(2013$, p. 173), et d'autre part, il affirme que cette dimension subjective se manifeste dans le fait que l'objet fabriqué porte la marque du sujet fabriquant, qui peut par conséquent s'y reconnaître, et donc, être reconnu (p. 174). C'est dire qu'il y a comme un double mouvement dans la dimension subjective du travail. Le travail est fait par quelqu'un et le travail fait quelqu'un. Je mets de moi-même dans mon œuvre, et mon travail met de lui-même en moi. Or, Saint-Exupéry insiste énormément sur ce deuxième mouvement : le travail fait devenir plus grand que soi, à savoir participant d'une communauté. Ce faisant, l'autre mouvement semble occulté : le fait que l'on retrouve de l'artisan dans son œuvre semble peu importer. Comme si, à insister lourdement sur le fait que l'objet n'est qu'un vecteur pour le don de soi de l'artisan, il en oubliant la consistance propre de l'œuvre. On voit bien la nécessité de prévenir de l'idolâtrie en atténuant le côté hypnotique de l'échange superficiel entre travail et pouvoir de consommation. Mais il ne faudrait pas rendre le diaphane cet échange superficiel, sous peine d'oublier la consistance propre du travailleur, son habileté, son art, sa «patte» et donc la fierté personnelle et la reconnaissance personnelle qui sont ainsi permises. L'habileté de l'artisan sert de véhicule au don de soi, mais elle mérité également une reconnaissance en tant que telle.

Enfin, un dernier point de bifurcation peut être soulevé, avec la même précaution que les précédents, puisqu'un pensée exprimée sur un mode poétique ne se prête pas au jeu de la controverse sans pouvoir s'y dérober à tout instant au nom de la stratification métaphorique des significations. Il 
s'agit de la confusion entre l'engendrement et la création. Les élaborations théologiques dans l'antiquité tardive autour de la controverse sur le statut de la deuxième personne de la trinité, « engendré, non pas créé » pourraient servir à thématiser cette limite. En effet, deux ordres bien distincts de causalité sont distinguables : l'engendrement met au monde un être de même nature que l'engendrant, selon une dynamique de fécondité, avec sa temporalité propre. Mais la reproduction n'est pas la production, la procréation n'est pas la création : en effet, le fabriqué n'est pas de même nature que le fabriquant, et aucune fécondité relationnelle n'est envisageable entre eux (au grand dam de Pygmalion qui se voit obligé de demander à la déesse d'instaurer une connaturalité). C'est plutôt le régime de l'efficacité, plus que celui de ce la fécondité, qui détermine la temporalité de la production. Dans la production, le fabriquant imprime une forme conçue en esprit dans une matière, tandis que dans la reproduction, c'est la forme même de l'engendrant qui transite dans l'engendré. Cette frontière fondamentale entre les deux domaines est estompée par le geste de Saint-Exupéry qui veut faire porter à la production les fonctions de la reproduction : demeurer une fois mort. Certes, l'auteur a de son côté la signification parturitionelle du mot travail, et on comprend que cette confusion permette de donner plus de sens au travail, en le plaçant sur une trajectoire existentielle. C'est pourquoi il ne faudrait prendre cette confusion que comme une invitation à l'unité de dimensions qu'on a tendance à séparer de façon excessive.

$\mathrm{Au}$ terme de cette réflexion apparait donc la limite du recours à une pensée exprimée de façon littéraire. Ces points de vigilance et ces limites de la pensée de Saint Exupéry n'empêchent pas de considérer son apport : concevoir un travail unifié dans ses trois dimensions, donc unifiant pour le travailleur.

On peut, pour conclure, mentionner une autre voie d'inspiration que pourrait apporter la pensée de Saint-Exupéry, tirée de tous ses écrits, y compris les plus littéraires. Cette piste que nous n'avons pas exploré, pourtant riche d'enseignement, relève de posture de leader. Dans Citadelle, Saint-Exupéry fait porter sa parole par un chef berbère. L'apport principal que Saint-Exupéry pourrait être d'inspirer un concept de leader débarrassé des oripeaux d'un humanisme discursif vide de contenu, si l'on en croit la critique de Dupuy contre le prêt-à-penser managérial, notamment autour d'expressions comme «l'homme doit être au centre de tout», (2015, p. 178). Se dessinerait alors une figure roborative du leader, décomplexé par apport à l'exercice d'une saine autorité conçue comme un service, réhabilitant la camaraderie tonique vécue par le pilote de guerre, à l'aise avec l'aspect stimulant de l'exigence. 


\section{Bibliographie}

Akerlof G. A., 1982, «Labor Contracts as Partial Gift Exchange », Quarterly Journal of Economies, ${ }^{\circ}$ 97, pp. 543-569.

Akerlof G. A., 1984, « Gift Exchange and Efficiency-Wage Theory : Four Views », American Economic Review, n 14, pp. 79-83.

Alter N., 2010, « Travail et déni du don », Revue du MAUSS, n 35, pp. 175-194.

Ambert N., 2001, « Le langage, l'écriture et laction dans Citadelle, ou l'art poétique de SaintExupéry », Études littéraires, vol. 33, p. 97-111.

Anthony P. D., 1980, «Le travail et la perte de sens », Revue internationale des sciences sociales, vol. XXXII, pp. 444-455.

Arendt H., 2014, La condition de l'homme moderne, Paris, Calmann-Levy.

Aristote, 1990, Ethique à Nicomaque, Paris, Vrin.

Aristote, 2002, Physiques, Paris, GF Flammarion.

Aristote, 2014, La politique, Paris, Vrin.

Babeau O. et Chanlat J.-F., 2011, « Déviance ordinaire, innovation et gestion. L'apport de Norbert Alter ", Revue française de gestion, $\mathrm{n}^{\circ} 210$, pp. 33-50.

Badiou A., 1998, Petit manuel d'inesthétique, Paris, Seuil.

Barrère A. et Martuccelli D., 2005, « La modernité et l'imaginaire de la mobilité : 'inflexion contemporaine », Cahiers internationaux de sociologie, $\mathrm{n}^{\circ} 118$, pp. 55-79.

Berthoin Antal A. et Fremeaux S., 2013, «Don gratuit, spiritualité au travail, sens au travail », Revue interdisciplinaire management et humanisme, $\mathrm{n}^{\circ}$ 8, pp. 3-18.

Bollnow O. F., 1981, «L'homme bâtisseur de cités », in Cahiers Saint-Exupéry, n², Paris, Gallimard, pp. 41-57.

Bowman M. J., 1965, « Exchange », in J. Gould, W.L. Kolb, A dictionary of social sciences, New York, Free press of Glencoe, pp. 247-248.

Caru A., Cova B., 2006, «Expériences de consommation et marketing expérientiel », Revue française de gestion, $\mathrm{n}^{\circ} 162$, pp. 99-113.

Cazeneuve J., 1968, Sociologie de Marcel Mauss, Paris, Puf

Chessel M.-E., 2012, «Au XXe siècle : vers la société de consommation contemporaine », in Histoire de la consommation, Paris, La Découverte.

Concialdi P., 2009, "Qu'importe le travail, pourvu qu'on ait le RSA », Travail, genre et sociétés, $\mathrm{n}^{\circ} 22$, pp. 177-182. 
Cugno A., 2006, « Le sens enfoui du travail », Projet, n² 291, pp. 59-66.

Darmangeat C., 2016, « Don, échange et autres transferts. Formes simples, hybrides et composées », L'Homme, n 217, pp. 21-43.

Davis J., 1996, «Exchange », in A. Kuper, J. Kuper, Social science encyclopedia, London, Routledge, pp. 274-275.

De Borchgrave R., 2006, Le philosophe et le manager, Bruxelles, De Boeck.

Drucker P. F., 1993, The practice of management, New York, Harper.

Dupuy J., 2015, La faillite de la pensée managériale, Paris, Seuil.

Godbout J., 1992, L'esprit du don, Paris, la Découverte.

Gomez P.-Y. 2015, « Sommes-nous des bisounours ?», in Gomez, P.-Y. et al., L'entreprise, une affaire de don, Bruyères-le-Châtel, Nouvelle Cité.

Heidegger M., 1986, Etre et temps, Paris, Gallimard.

Kerneff J., 2001, «L'éternité chez Saint-Exupéry », in Le Hir G., Antoine de Saint-Exupéry, Etudes littéraires, n³3, pp. 83-94.

Leclercq-Vandelannoitte A., Isaac H., 2013, «Technologies de l'information, contrôle et panoptique: Pour une approche deleuzienne ", Systèmes d'information \& management, $\mathrm{n}^{\circ}$ 18(2), pp. 9-36.

Leclercq-Vandelanoitte A., 2011, «Organizations as Discursive Constructions: A Foucauldian Approach », Organization Studies, n 32(9), pp. 1247-1271.

Maréchal J.-P., 2001, « Le travail et l'analyse économique », Études, Tome 395, pp. 455-465.

Marx K., 1985, Le Capital, livre I, Paris, Flammarion.

Mauss M., 2012, Essai sur le don. Forme et raison de l'échange dans les sociétés archaïques, Puf.

Méda D., 2004, Le travail, Paris, Puf.

Méda D., 2010, «Comment mesurer la valeur accordée au travail ?», Sociologie, Vol. 1, pp. 121-140.

Méda D., 2010, «Est-il possible de libérer le travail ? », Le Philosophoire, n 34, pp. 55-69.

Michel D.-A., 2012, «Le management, nœud de contradictions », L'Expansion Management Review, $\mathrm{n}^{\circ} 145$, p. 3-3.

Niebuhr R., 1935, An interpretation of Christian ethics, New York, Harper.

Noel C. et Le Loarne S., 2007, « Libre pensée du travail et organisation responsable. Ce que nous apprend La philosophie de Simone Weil », Revue de philosophie économique, $\mathrm{n}^{\circ} 8$ (2), pp. 99-121. 
Perez R., 2005, «Quelques réflexions sur le management responsable, le développement durable et la Responsabilité sociale de l'entreprise », La Revue des Sciences de Gestion, $\mathrm{n}^{\circ}$ 212, pp. 29-46.

Pesqueux Y., 2011, «La responsabilité sociale de l'entreprise (RSE) comme discours ambigu », Innovations, n³4, pp. 37-55.

Proudhon P.-J., 1873, Qu'est ce que la propriété [en ligne], users.teledisnet.be (consulté le 3 septembre 2013).

Quesnel M., 1989, «Lire "Citadelle” », in Cahiers Saint-Exupéry, n³, Paris, Gallimard, pp. 55-122.

Ravoux J.-P., 1980, «Travail, échange et liens », in Cahiers Saint-Exupéry, n 3, Paris, Gallimard, pp. 106-134.

Ricœur P., 1990, Soi-même comme un autre, Paris, Seuil.

Sabot P., 2002, Philosophie et littérature : approches et enjeux d'une question, Paris, PUF.

Saint-Exupéry A., 1946, Le Petit Prince, Paris, Gallimard.

Saint-Exupéry A., 1948, Citadelle, Paris, Gallimard.

Saint-Exupéry A., 1994, « Carnets », in Euvres complètes, I, Paris, Gallimard.

Saint-Exupéry A., 2015, « Messages aux jeunes américains », in F.-X. Bellamy, A la jeunesse, Paris, Librio, pp. 67-70.

Salzmann Y., 2000, Sartre et l'authenticité: vers une éthique de la bienveillance réciproque, Genève, Labor et fides.

Sartre J.-P., 1983, Cahiers pour une morale, Paris, Gallimard.

Tirole J., 2009, « Motivation intrinsèque, incitations et normes sociales », Revue économique, vol. 60, pp. $577-589$. 